\title{
NLO quark mass effects in polarized DIS
}

\author{
1. AKUSHEVICH *) \\ North Carolina Central University, Durham, NC 27707, USA \\ and TJNAF, Newport News, VA 23606, USA \\ A. ILyichev, N. SHumeiko \\ National Centre for Particle and High Energy Physics, \\ Bogdanovich str. 153, 220040 Minsk, Belarus
}

The method originally developed for the exact calculations in QED theory is applied for the calculation NLO effects in QCD Compton processes. QCD corrections to the structure functions and sum rules are obtained. Different interpretations of the NLO effects due to finite quark mass are discussed.

\section{Introduction}

Up to know a lot of articles dedicated to NLO QCD corrections to polarized DIS have been published. In most of them all calculations are performed in the assumption that quark mass is equal to zero (see [1,2] and review [3]). At the same time there are some works whose authors estimate a finite quark mass effects both for unpolarized [4] and polarized structure functions $[5,6,7,8]$. The main difference of these two approaches consists in the method of tending quark mass to zero and procedure of integration over the phase space of gluon emission. So in the massless one fermion mass is equal to zero before integration while in the second case this quantity tends to zero after integration and survives in LO terms only.

Notice that QCD and QED RC having different origins on the one-loop level possess some common features. If our consideration is restricted to the so-called QCD Compton process then both these corrections will be described by the identical sets of Feynman graphs. The transition from the strong radiative effects to the electromagnetic ones could be performed by the following replacement:

$$
\frac{4}{3} \alpha_{\varepsilon} \rightarrow e_{q}^{2} \alpha_{Q E D}
$$

In the present report we investigate QCD Compton process for polarized nucleon target by straightforward method developed for exact calculations $[9,10,11,12,13]$ in QED theory for massive polarized fermions but never used for QCD ones. We estimate the influence of finite quark mass effects on the value of the polarized sum rules and discuss some different interpretations of the first moment of the polarized structure functions $g_{1}$.

*) On leave of abeence from the National Center for Particle and High Energy Physics, 220040 Minsk, Belarus. 


\section{Method of Calculation and Main Results}

The lowest-order one-loop RC to the hadronic current consists of two parts whose contributions are calculated in a different way: $W_{\mu \nu}^{1-l o o p}=W_{\mu \nu}^{R}+W_{\mu \nu}^{V}$. The first one appears from a gluon emission and requires the integration over its phase space, while the second part comes from a gluon exchange graph and reads

$$
\begin{aligned}
W_{\mu \nu}^{V}= & \frac{4}{3} \frac{\alpha_{s}}{\pi} \sum_{q}\left[-2\left(\mathcal{P}^{I R}+\ln \frac{m_{q}}{\mu}\right)\left(l_{q}-1\right)-\frac{1}{2} l_{q}^{2}+\frac{3}{2} l_{q}\right. \\
& \left.-2+\frac{\pi^{2}}{6}\right] W_{\mu \nu}^{0 q}+W_{\mu \nu}^{A M M},
\end{aligned}
$$

where $W_{\mu \nu}^{0 q}$ is a contribution of $q$-quark to the hadronic tensor on the Born level, and $W_{\mu \nu}^{A M M}$ is the quark anomalous magnetic moment. The pole term which corresponds to the infrared divergence is contained in $\mathcal{P}^{I R}$. The arbitrary parameter $\mu$ has a dimension of a mass.

Both of these contributions include the infrared divergences, which have to be careful considered in order to be canceled. Like QED we use the identity

$$
W_{\mu \nu}^{R}=W_{\mu \nu}^{R}-W_{\mu \nu}^{I R}+W_{\mu \nu}^{I R}=W_{\mu \nu}^{F}+W_{\mu \nu}^{I R} .
$$

Here $W_{\mu \nu}^{F}$ is finite for $k \rightarrow 0$, and $W_{\mu \nu}^{I R}$ is the infrared divergent part of $W_{\mu \nu}^{R}$. Using the dimensional regularization scheme the latter can be given in the form

$$
\begin{array}{r}
W_{\mu \nu}^{I R}=\frac{4}{3} \frac{\alpha}{\pi} \sum_{q}\left[2\left(\mathcal{P}^{I R}+\ln \frac{m_{q}}{\mu}\right)\left(l_{q}-1\right)+l_{q} l_{v}+\frac{1}{2} l_{q}^{2}\right. \\
\left.-\frac{1}{2} l_{v}^{2}-\frac{3}{4} l_{q}-\frac{7}{4} l_{v}+\frac{3}{4}-\frac{\pi^{2}}{3}\right] W_{\mu \nu}^{0 q}
\end{array}
$$

where $l_{q}=\log \left(Q^{2} / m_{q}^{2}\right), l_{v}=\log ((1-x) / x)$. The sum of $W_{\mu \nu}^{I R}$ and $W_{\mu \nu}^{V}$

$$
\begin{aligned}
W_{\mu \nu}^{I R}+W_{\mu \nu}^{V} & =\frac{2}{3} \frac{\alpha_{s}}{\pi} \sum_{q}\left[2 l_{q} l_{\nu}-l_{v}^{2}+\frac{3}{2} l_{q}-\frac{7}{2} l_{v}-\frac{5}{2}-\frac{\pi^{2}}{3}\right] W_{\mu \nu}^{0 q}+W_{\mu \nu}^{A M M} \\
& =\frac{2}{3} \frac{\alpha_{s}}{\pi} \sum_{q} \delta_{q} W_{\mu \nu}^{0 q}+W_{\mu \nu}^{A M M}
\end{aligned}
$$

is infrared free.

In order to extract some information about QCD contribution to the polarized structure functions, the integration in $W_{\mu \nu}^{F}$ over the gluon phase space should be performed without any assumptions about the polarization vector $\eta$. So the technique of tensor integration have to be applied in this case. Since the result of the analytical integration has the same tensor structure as the usual hadronic tensor in polarized DIS, the coefficients in front of the corresponding tensor structures (like $g_{\mu \nu}, p_{\mu} p_{\nu} \ldots$ ) can be interpreted as one-loop QCD contributions to the corresponding structure functions. 
Thus the QCD-improved structure functions $F_{1,2, L}$ and $g_{1}$ read

$$
\begin{gathered}
F_{1}\left(x, Q^{2}\right)=\frac{1}{2 x}\left[F_{2}\left(x, Q^{2}\right)-F_{L}\left(x, Q^{2}\right)\right], \quad F_{2}\left(x, Q^{2}\right)=x \sum_{q} e_{q}^{2} f_{q}\left(x, Q^{2}\right), \\
F_{L}\left(x, Q^{2}\right)=\frac{4 \alpha_{s}}{3 \pi} x \sum_{q} e_{q}^{2} \int_{x}^{1} d z f_{q}(x / z), g_{1}\left(x, Q^{2}\right)=\frac{1}{2} \sum_{q} e_{q}^{2} \Delta f_{q}\left(x, Q^{2}\right),
\end{gathered}
$$

and $g_{2}$ looks like

$$
\begin{aligned}
g_{2}\left(x, Q^{2}\right) & =\frac{\alpha_{q}}{6 \pi} \sum_{q} e_{q}^{2}\left\{\left(1-2 l_{q}-\ln (1-x)\right) \Delta f_{q}(x)+\int_{x}^{1} d z\left[\left(4 l_{q}\right.\right.\right. \\
& \left.\left.\left.-4 \log z(1-z)-12-\frac{1}{(1-z)}\right) \Delta f_{q}(x / z)+\frac{\Delta f_{q}(x)}{(1-z)}\right]\right\}
\end{aligned}
$$

where the $Q^{2}$-dependent unpolarized and polarized parton distributions are defined as

$$
\begin{array}{r}
f_{q}\left(x, Q^{2}\right)=\left(1+\frac{2 \alpha_{z}}{3 \pi} \delta_{q}\right) f_{q}(x)+\frac{2 \alpha_{g}}{3 \pi} \int_{x}^{1} \frac{d z}{z}\left[\left(\frac{1+z^{2}}{1-z}\left(l_{q}-\log z(1-z)\right)\right.\right. \\
\left.\left.-\frac{7}{2} \frac{1}{1-z}+3 z+4\right) f_{q}\left(\frac{x}{z}\right)-\frac{2}{1-z}\left(l_{q}+\log \frac{z}{1-z}-\frac{7}{4}\right) f_{q}(x)\right] \\
\Delta f_{q}\left(x, Q^{2}\right)=\left(1+\frac{2 \alpha_{q}}{3 \pi} \delta_{q}\right) \Delta f_{q}(x)+\frac{2 \alpha_{q}}{3 \pi} \int_{z}^{1} \frac{d z}{z}\left[\left(\frac{1+z^{2}}{1-z}\left(l_{q}-\log z(1-z)\right)\right.\right. \\
\left.\left.-\frac{7}{2} \frac{1}{1-z}+4 z+1\right) \Delta f_{q}\left(\frac{x}{z}\right)-\frac{2}{1-z}\left(l_{q}+\log \frac{z}{1-z}-\frac{7}{4}\right) \Delta f_{q}(x)\right],
\end{array}
$$

and $\delta_{q}$ can be found in (3).

It has to be noted that our formulae $(4,5,6)$ are in an agreement with ones obtained earlier. The unpolarized structure functions coincide with $(2.24,2.49)$ from [4], $g_{1}\left(x, Q^{2}\right)$ corresponds to the expression (13) in [5]. At last $g_{2}\left(x, Q^{2}\right)$ can be considered with the sum (18) and (19) from [6].

After integration of the QCD-improved structure functions over the scaling variable $x$ it could be seen that QCD RC to the first moment of the unpolarized structure functions as well as $g_{2}$ have the identical values both for massive and massless quark approaches. However the value of QCD-correction to the first moment of $g_{1}$ depends on the scheme. It was calculated:

$$
\int_{0}^{1} g_{1}\left(x, Q^{2}\right)=\left(1-C_{g 1} \frac{\alpha_{g}}{\pi}\right) \int_{0}^{1} d x g_{1}^{0}(x),
$$

where $C_{g 1}=1$ for massless and $C_{g 1}=5 / 3$ for massive one. 
It should be noted that this result has been found in [5]. However the authors considered this effect as an unphysical one and suggested the factorization mass procedure in order to remove it.

This correction was discussed in ref. [7] from the partonic and in [8] from the OPE point of view. The classical result for first order correction to Bjorken sum rule is probably reconstructed if the correction to $g_{a}$ is also taken into account. Also we would like to note that the region of soft quarks appearing under integration region requires of careful separate consideration within QPM calculations. It is taken into account for QCD [7], but usually not for QED hadronic corrections.

We thank O. Teryaev for valuable discussions. One of us (I. A.) thanks the US Department of Energy for support under contract DE-AC05-84ER40150.

\section{References}

[1] P. Ratcliffe: Nucl. Phys. B223 (1983) 45.

[2] M. Glück, E. Reya and M. Stratmann, W. Vogelsang: Phys. Rev. D 53 (1996) 4775.

[3] B. Lampe, E. Reya: MPI-PHT-98-23 Apr 1998. 134pp.

[4] B. Humpert and W.L. van Neerven: Nucl. Phys. B 184 (1981) 498.

[5] R. Mertig and W.L. van Neerven: Z. Phys. C 60 (1993) 489.

[6] G. Altarelli, B. Lampe, P. Nason and G. Ridolfi: Phys. Lett B 334 (1994) 184.

[7] O. Teryaev: in Contribution to International Symp. Dubna Deutreron '95, Dubna Russia 1995, hep-ph/9508374.

[8] O. Teryaev, O. Veretin: hep-ph/9602362.

[9] D. Bardin, N. Shumeiko: Nucl. Phys. B 127 (1977) 242.

[10] I. Akushevich and N. Shumeiko. J. Phys: G 20 (1994) 513.

[11] I. Akushevich, A. Ilyichev and N. Shumeiko: J. Phys. G 24 (1998).

[12] A. Akundov, D. Bardin, L. Kalinovskaya, T.Rieman: Fortsch. Phys. 44 (1996) 373.

[13] D. Bardin, L. Kalinovskaya: DESY-97-230 Dec 1997. 54pp. 